\title{
Jiří Gruša - ein mitteleuropäischer Dichter, Politiker und Kulturvermittler
}

Renata Cornejo, Jiří Gruša : un poète, homme politique et passeur culturel en Europe centrale

Renato Cornejo, Jiří Gruša: A Central European poet, politician and cultural mediator

\section{Renata Cornejo}

\section{OpenEdition}

\section{Journals}

Édition électronique

URL : https://journals.openedition.org/austriaca/747

DOI : 10.4000 /austriaca.747

ISSN : 2729-0603

\section{Éditeur}

Presses universitaires de Rouen et du Havre

\section{Édition imprimée}

Date de publication : 1 décembre 2019

Pagination : 163-173

ISBN : 979-10-240-1454-8

ISSN : 0396-4590

\section{Référence électronique}

Renata Cornejo, „Jiř́ Gruša - ein mitteleuropäischer Dichter, Politiker und Kulturvermittler", Austriaca [Online], 88-89 | 2019, Online erschienen am: 31 Dezember 2020, abgerufen am 24 Oktober 2022. URL: http://journals.openedition.org/austriaca/747 ; DOI: https://doi.org/10.4000/austriaca.747 


\section{Jiří Gruša - ein mitteleuropäischer Dichter, Politiker und Kulturvermittler}

\section{Die Brüche und Umbrüche in Grušas Leben (nicht nur mit einer 8)}

Das Leben und Wirken des am 10. November 1938 in Pardubice/Pardubitz - einem Tag nach der Reichskristallnacht - geborenen Schriftstellers Jiří Gruša steht im Vorzeichen der für Mitteleuropa geschichtsträchtigen Jahre mit der Zahl 8 am Ende: 1938 wurde der erste freiheitlich-demokratische und soziale Rechtsstaat nach westlichem Vorbild im mitteleuropäischen Raum, die am 28. Oktober 1918 gegründete Tschechoslowakische Republik, zum politischen Spielball der Großmächte Großbritannien, Italien und Frankreich. Durch das am 30. September 1938 verabschiedete Münchner Abkommen wurde die junge demokratische Republik zum Abtreten ihres Schutzwalles und sonstiger Grenzgebiete an Hitlers „Drittes Reich“ gezwungen, im März des darauffolgenden Jahres besetzten deutsche Truppen die sog. Rest-Tschechei und es wurde das Protektorat Böhmen und Mähren unter deutscher Hoheit ausgerufen. Damit hörte die Tschechoslowakei zunächst einmal auf zu existieren. Für ihre "Wiederauferstehung" nach dem Ende des Zweiten Weltkrieges in ihren ursprünglichen Grenzen (ausgenommen die an die Sowjetunion abgetretene Karpatenukraine) bedeutete das Jahr 1948 den nächsten wichtigen geschichtlichen Umbruch. Nach dem Rücktritt der nichtkommunistischen Mitglieder der tschechoslowakischen Regierung akzeptierte der damalige Staatspräsident Eduard Beneš die neue, kommunistisch dominierte Regierung, die er am 25. Februar 1948 offiziell vereidigte. Mit diesem Tag, der in die sozialistischen Geschichtsbücher als „Der siegreiche Februar" eingegangen ist, übernahm die Kommunistische Partei der Tschechoslowakei (KSČ) kampflos die Vorherrschaft und begann die weitere innen- sowie außenpolitische Entwicklung des Landes maßgeblich zu bestimmen. $\mathrm{Zu}$ diesem Zeitpunkt ist Jiří Gruša gerade 10 Jahre alt. 
Aus einer Beamtenfamilie katholischen Glaubens und kritischer Gesinnung stammend studierte er nach dem Abschluss des Gymnasiums Geschichte und Philosophie an der Karlsuniversität Prag und trat bereits Anfang der 1960er Jahre als Mitbegründer der ersten nichtkommunistischen Zeitschrift Das Gesicht (1963) in Erscheinung. Schon ein Jahr davor publizierte er seinen ersten Gedichtband Torna (Der Tornister) und betrat somit die tschechische Literaturszene zunächst als Dichter, später auch als erfolgreicher Prosaautor. Seiner kritischen Einstellung kam die politische Auflockerung und die Reformbewegung der 60er Jahre entgegen, die einen „Sozialismus mit menschlichem Antlitz" anstrebte und mit der Besetzung der Tschechoslowakei durch die „verbündeten Truppen“ des Warschauer Paktes gewaltsam unterbrochen wurde - am 21. August 1968. Diese 8, die Niederschlagung des Prager Frühlings, sollte für Gruša die wichtigste in seinem Leben werden, denn während der darauffolgenden sog. Normalisierungszeit - Zeit der Wiederherstellung der politischen und gesellschaftlichen Verhältnisse vor 1968 - erhielt er wegen seiner schriftstellerischen Tätigkeit bald ein Berufsverbot. In den 70er Jahren wurde er wegen der Veröffentlichung seiner Werke in Samisdat-Zeitschriften (illegal verbreitete Manuskripte im Selbstverlag) auch strafrechtlich verfolgt (offiziell wegen dem pornographischen Inhalt seines Romans Mimner aneb hra o smradocha, dt. Mimner oder das Tier der Trauer). Es überrascht in diesem Zusammenhang wenig, dass Jiří Gruša die Charta 77, eine in den wichtigsten europäischen Zeitungen veröffentlichte Petition der Regimegegner (sog. Dissidenten), unterzeichnet hatte und demzufolge weiteren Repressionen ausgesetzt war - u.a. wurde er wegen seinem im kanadischen Exilverlag publizierten Roman Dotazník (Der Fragebogen) zur mehrmonatigen Gefängnisstrafe verurteilt, die dank der internationalen Proteste auf zwei Monate verkürzt werden konnte. Zur schicksalhaften und folgenschweren Entscheidung kam es seinerseits, als er sich entschieden hat, trotz der Warnungen seiner Freunde sowie eigener Befürchtungen (1978 wurde während eines arbeitsmäBig bedingten Aufenthaltes in Wien der politisch unbequeme Schriftsteller Pavel Kohout ausgebürgert) nahm er das Angebot eines Stipendienaufenthalts in den USA im Jahr 1980 wahr und stand in Kürze, wie befürchtet, im Ausland plötzlich staatenlos. Als ein in der BRD inzwischen bekannter Autor (sowohl durch die Übersetzungen seiner Romane ins Deutsche als auch durch seine Inhaftierung und vorzeige Freilassung aus dem Gefängnis, die v. a. die Intervention von Heinrich Böll erwirkte) entschied sich Gruša für das Verbleiben in der BRD - 
eine durchaus logische Entscheidung, denn die deutsche Sprache und Kultur war ihm keineswegs fremd: Mit der deutschsprachigen Literatur machte er sich schon während seines Hochschulstudiums bekannt, in der Familie seiner ersten Frau (Tochter des Prager führenden Germanisten Eduard Goldstücker) sprach man deutsch, vom Deutschen ins Tschechische hat er selbst bereits einige Dichtungen erfolgreich übertragen (z. B. R. M. Rilke). So fiel die Wahl auf die BRD (Bonn), wo er mit einigen Unterbrechungen bis zu seinem Tod 2011 als Dichter, Denker und Politiker gewirkt hat.

Eine weitere Zäsur in seinem Leben bedeutete das Jahr 1989, das ihm einerseits unerwartet den frühzeitigen und ungeklärten Tod seines einzigen, in der Tschechoslowakei verbliebenen Sohnes mitbrachte und andererseits, genauso unerwartet, den Zusammenbruch des sozialistischen Regimes in der Tschechoslowakei und damit die Möglichkeit, in die vor Jahren ungewollt verlassene Heimat wieder legal einzureisen - leider zu spät, um dem Begräbnis des verstorbenen Sohnes beiwohnen zu können.

\section{Jiří Gruša als Dichter}

Jiři Gruša begann seine schriftstellerische Laufbahn als Dichter, er betrat die tschechische Literaturszene 1962 mit einem Gedichtband (Der Tornister), die deutsche Literaturszene - nach einem sehr schwierigen, mit einem psychischen und physischen Zusammenbruch verbundenen Sprachwechsel - fast dreißig Jahre danach (1991) ebenfalls mit einem Gedichtband (Der Babylonwald). Das ihm der späte Sprachwechsel (mit 50 Jahren) viel abverlangte und fast sein Leben kostete, ist näher in seiner 3. Dresdner Poetik-Vorlesung nachzulesen ${ }^{1}$. Das „Fallen“ (tsch. padání) in die neue, für ihn fremde Sprache zählt Gruša zu einer der sieben axiomatischen Absurditäten seines Lebens ${ }^{2}$, mit denen er merkwürdige, mit-

1. Vgl. Jiř́ Gruša, „Der Fall oder das Präsens als Zeitform der Lyrik“, in Jiř́ Gruša, Das Gesicht - der Schriftsteller - der Fall. Vorlesungen über die Prätention der Dichter, die Kompetenz und das Präsens als die Zeitform der Lyrik. Dresden, Thelem Verlag, 2000, S. 39-56 oder auch Renata Cornejo, „Der Fall Jiří Gruša und sein ,Fallen' in die deutsche Sprache", in Zeitschrift für interkulturelle Germanistik (ZiG), Jg. 6, H. 2/2015, S. 103-116.

2. Als die erste „axiomatische Absurdität" seines Lebens bezeichnet Gruša die Tatsache, dass er in seiner Geburtsstadt Pardubice als kleiner Junge versucht hat aus dem Kloster der Salesianer Schauspieltexte zu entwenden, aber auch, dass er an diesen Ort nach fast 40 Jahren zurückgekehrt ist, um seine von der tschechoslowakischen Staatssicherheit geführte Akte einzusehen. Die zweite „axiomatische Absurdität“ sieht er darin, dass er gemeinsam mit seinem Freund Jiř́ Pištora bei einer Schauspielaufführung in Pardubice das erste Mal in seinem Leben vor einem Publikum sprach und an Pištoras Grab, wieder 
einander im Zusammenhang stehende Begebenheiten in seinem Leben bezeichnet. Das „Fallen“ in die neue Sprache, die vierte axiomatische Absurdität, hält er zugleich für den „Wappenspruch ${ }^{3 “}$ für seine Poetik, die er als einen schmerzvollen Lernprozess verstehen will:

In dem zuletzt gedruckten Gedichtband Cvičení mučení erschien es [das Gedicht Padáni] im Januar 1969, kurz vor dem endgültigen Garaus. Es folgten 21 Jahre des Stumm-Seins in patria. Es war also etwas wie ein vorverlegter Nachlaß und eine Prognose zugleich. Der Buchtitel war eine Anspielung auf das Sprichwort „Ohne Fleiß kein Preis“. Auf Tschechisch lautet das: Ohne Pein kein Lernen. Pein lernen - so ließe sich das vielleicht eindeutschen. Und damals deutete es auf einen aufkommenden Lernprozeß hin - mit den Peinigern leben zu müssen. Die Sicht war richtig, nur die Länge der Lehrzeit habe ich unterschätzt ${ }^{4}$.

Der „peinvolle“ Lernprozess als selbst „ergründete Grundlage“ der Poetik erhält in diesem Zusammenhang eine vorahnende Doppelbedeutung und wird zum Sinnbild des schmerzvoll erfahrenen Selbstverlustes und der doppelten (Nicht)Existenz des Dichters - zunächst in seiner Muttersprache in der politisch restriktiven Tschechoslowakei, in der die Stimme eines unbequemen Dichters zum Schweigen gebracht wird, später in seiner neuen, deutschen Sprache, die dem Dichter als Literatursprache in ihrer Vollkommenheit für immer verschlossen und unerreichbar zu bleiben scheint. Dieser Zwiespalt und zeitweilige Verzweiflung des Autors, die ihren poetischen Ausdruck im bereits erwähnten Gedicht Padání und seiner graphischen „Übersetzung “ in eine Zeichensprache findet und schließlich zur Überwindung einer vorübergehender „Verstummung" des Dichters (ermöglicht durch vorübergehende Erblindung) führt, sind auch im Gedicht Wortschaft präsent, das 1994 im Gedichtband Wandersteine veröffentlicht wurde:

in Pardubice, seine letzte Rede vor einem Publikum gehalten hatte, bevor ihm das öffentliche Redeverbot erteilt wurde. Die dritte „axiomatische Absurdität“ bezieht er auf den unterschiedlichen Genus des Todes in der tschechischen und der deutschen Sprache (im Tschechischen ist der Tod weiblich), die vierte auf das „Fallen“ als Chiffre der eigenen Poesie. Als sechste „Axio-Absurdität“ bezeichnet er seinen Auftritt im Dezember 1989 im Prager Nationaltheater, als er sich ertappte, „flammende Worte“von der Bühne „zu schleudern“. Die siebente bedeutete für ihn das Gedicht an seinen Sohn Martin, das er nach seiner Rückkehr nach Prag in deutscher Sprache auf einem Zettel in einem Prager Café niederschrieb - Martin starb mit 23 Jahren, genauso „alt“ wie Grušas tschechisches Schreiben (vgl. Jiří Gruša, Das Gesicht - der Schriftsteller - der Fall, S. 10, 17, 40, 43, 52, 53, 54).

3. Ebd., S. 39-56, S. 43.

4. Ebd., S. 43. 


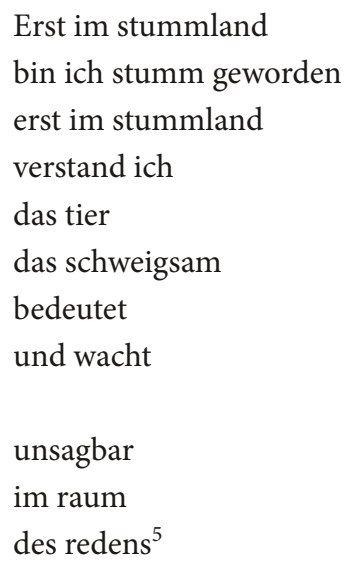

Einerseits thematisiert das Gedicht die Sprachlosigkeit eines zum Schweigen gebrachten lyrischen Ich, das sich in einem "stummland“ befindet und fungiert als Chiffre für die Spaltung in die offizielle (wortlaute) und nicht offizielle (wortlose) Literatur in der Tschechoslowakei der Normalisierungszeit, wenn die Entstehungszeit des Gedichts (1980er Jahre) berücksichtigt und somit der aktuelle „politische Kontext“ herangezogen wird. Genauso aber kann die Wortbildung „stummland" auch als eine Chiffre für das Land gelesen werden, in welches das lyrische Subjekt gegen seinen Willen versetzt und so seiner Dichtersprache beraubt wurde, wenn das Gedicht „biographisch“ auf die Migrationserfahrung des Autors hin kontextualisiert wird. In dem Fall funktioniert das Wort „stummland“ als Sprachspiel und kann als die wörtliche Übersetzung des Wortes „Německo“ (Deutschland) aus dem Tschechischen (němý - stumm, Německo - stummland) dechiffiert werden. Den erzwungenen und deswegen schmerzvollen Verlust der Heimat und Sprache sowie das Ringen um deren Wiedergewinnung "ein meeresufer suchend, wo man belaubt ${ }^{6 \text { " }}$ - wurde zum Hauptthema seiner beiden auf Deutsch verfassten Gedichtbände, wie bereits durch die symbolträchtigen Titel signalisiert wird. Der Babylonwald (1991) lässt sich, wie die Metapher "stummland“, in beide Richtungen lesen als Chiffre sowohl für die babylonische Gefangenschaft, aus der sich das unterjochte Volk einmal befreien wird (politischer Kontext) als auch für

5. Jiři Gruša, „Wortschaft", in Ders., Wandersteine. Gedichte. Stuttgart, Deutsche Verlags-Anstalt, S. 40.

6. Jiř́ Gruša, „Babylon - der Wald in Ensko“, in ders. Der Babylonwald. Gedichte, Stuttgart, Deutsche Verlags-Anstalt, 1991, S. 23. 
die göttliche Verwirrung der Sprachen und das sich Nicht-VerstehenKönnen (Migrationskontext). Die zweite Deutungsebene wird im Titel Wandersteine weiter verstärkt und bildhaft aufgebaut, indem die wandernden Steine für den inneren Zwiespalt des lyrischen Subjekts sowie seinem Wunsch nach dessen Überwindung widerspiegeln. So stehen die Wandersteine leitmotivisch für Gratwanderung zwischen BleibenWollen und Gehen-Müssen, zwischen Beständigkeit und Bewegung, Entwurzelung und Verankerung, zwischen der rückwärtsgewandten Trauer und der zukunftsorientierten Hoffnung, zwischen zwei Sprachen und Kulturen?

\section{Jiří Gruša als Politiker und Kulturvermittler}

Die Erfahrung des Sprachwechsels und damit zusammenhängender Machtlosigkeit angesichts des Verlustes der Dichtersprache - des eigentlichen Instruments und Ausdrucksmittels - prägte maßgeblich nicht nur Grušas philosophisches und künstlerisches Denken, sondern auch sein politisches Handeln. Gruša selbst versteht sein literarisches Werk durchaus politisch, auch wenn er explizit kaum auf die politischen Zustände in der damaligen Tschechoslowakei Bezug nimmt. Nach Gruša lässt sich kein literarisches Werk von seinem gesellschaftlichen Kontext, in dem es entstanden ist und auf welchen es reagiert, trennen. Diesen Zusammenhang zwischen Text und Kontext bezeichnet er als „das vitale Gleichheitszeichen zwischen der Vita und dem Wort ${ }^{8 *}$. Dass seine, auf den ersten Blick apolitischen Gedichte auch eine „politische“ Dimension haben, ist besonders in seinen „Böhmen-Gedichten“ ablesbar. Schon Shakespeare wusste, dass Böhmen am Meer liegt. Ingeborg Bachmann verlieh diesem Motiv in den 60er Jahren in ihrem berühmten Gedicht Böhmen liegt am $\mathrm{Meer}^{9}$ eine neue Dimension, als sie Böhmen zur utopischen Heimat der Vaganten aller Welt und zum Land der eigenen Wahl stilisierte, wo man dem Dichter seine innere Freiheit nicht strittig machen kann.

Auch Gruša greift dieses Motiv in seinen Gedichten auf: Das Bachmannsche Böhmen am Meer wird bei ihm zum sicheren Hafen für

7. Vgl. Renata Cornejo, Heimat im Wort. Zum Sprachwechsel der deutsch schreibenden tschechischen Autorinnen und Autoren nach 1968. Eine Bestandsaufnahme, Wien, Praesens Verlag, 2010, S. 188f.

8. Gruša, Das Gesicht - der Schrifsteller - der Fall, S. 31.

9. Vgl. Ingeborg Bachmann, „Böhmen liegt am Meer“, in dies., Werke I. Gedichte, Hörspiele, LIbretti, Übersetutzungen, München, Piper Verlag, 1982, S. 167. 
alle Gestrandeten und Schiffbrüche, wo die Möwen "gellend / willkommen heißen / hergeschwommenes /10". Es ist ein Ort, an dem die innere Zerrissenheit des lyrischen Ich endlich überwunden werden kann, indem es zu seinen Wurzeln, „wo man uns zeugt“, zurückkehrt und dem verlorenen Sohn wieder begegnen kann: „Der emsige ozean / unterbricht mich nicht / als ich ihn grüße / den sohn“. Gewidmet ist das Gedicht dem verstorbenen Sohn Martin, der „dort“ 1989 „gelandet $^{11 \text { " }}$ ist, so dass das Meer primär als Metapher für den ewigen Kreislauf, Leben und Tod, die Wiedergeburt und den Fluss unseres Lebens fungiert, doch der Bachmannsche Entwurf des utopischen Freiheitskonzepts schwingt eindeutig im Text mit und verweist auf die mit dem Prager Frühling verbundenen Freiheitshoffnungen und Aufbruchsstimmung. Diese wird auch zur zentralen Aussage in einem weiteren Böhmen-Gedicht: Der Ruf „über das tal zu Jesinka / zu höllenhexen“ ist unüberhörbar und zugleich eine Bitte des lyrischen Ich „dich herbeizubringen / der sich schon aufhebt / sich auf den Weg macht / und noch in den / sichtbaren hügeln / zur schneewehe wird ${ }^{12 “}$. Diese, ebenfalls ein Gedicht von Ingeborg Bachmann ${ }^{13}$ evozierenden Verse nehmen das Leitmotiv des Gedichtbandes, die wandernden Steine, wieder auf, doch zugleich wird durch den Gedichttitel Böhmen - ein Wintermärchen auch eine weitere Deutungsmöglichkeit eröffnet, da damit explizit Bezug auf Heinrich Heines politische Satire Deutschland - ein Wintermärchen genommen wird. Das Böhmen-Motiv wird nun nicht nur biographisch, sondern auch politisch verortet (Identifikation mit der Figur eines Migranten, der sich durch seine unfreie Heimat auf Wanderschaft begibt und deren politische und soziale Missstände kritisiert).

Dass Jiří Gruša die Zustände in seinem Heimatland immer am Herzen lagen, schimmert nicht nur in seinen Gedichten durch, sondern bezeugt auch seine politische und diplomatische Laufbahn nach dem Zusammenbruch des Ostblocks 1989. Nach dem Fall des „eisernen Vorhangs" stand er als einer der ersten an der deutsch-tschechischen Grenze und konnte es kaum erwarten, sich aktiv an dem gegenseitigen

10. Jiří Gruša, „Böhmen am Meer“, in Ders., Der Babylonwald. S. 65.

11. Ebd.

12. Jiří Gruša, „Böhmen - ein Wintermärchen“, in ders., Wandersteine, S. 70.

13. Vgl. das Gedicht Die gestundete Zeit („Es kommen härtere Tage. / Die auf Wiederruf gestundete Zeit / wird sichbar am Horizont“ (Ingeborg Bachmann, Die gestundete Zeit, München, R. Piper Verlag, 1957). 
Auf- und Ausbau der tschechisch-deutschen Beziehungen zu beteiligen und seine Erfahrungen einzubringen. Dieser Aufgabe konnte er in den Jahren 1990-1998 in der Funktion des tschechischen Botschafters in Bonn und von 1998 bis 2004 in Wien nachgehen, in den Jahren 2005 bis 2009 war er als Direktor der Diplomatischen Akademie Wien tätig. Im Unterschied zur erfolgreichen Diplomatenkarriere im Ausland (Bonn und Wien) stießen seine innenpolitischen Bemühungen auf wenig Anerkennung. Im Jahr 1997 bekleidete er zwar den Posten des tschechischen Bildungsministers in der Regierung von Václav Klaus, doch nur einige Monate (Juni - November). Seine misslungenen Versuche, sich 1996 als regionaler Politiker im Wahlkreis Cheb nützlich zu machen sowie seine Enttäuschung über das Misstrauen und die Vorurteile gegenüber einem Tschechen, der aus Deutschland kam, thematisiert er näher in seinem Essayband Glücklich heimatlos. Einblicke und Rückblicke eines tschechischen Nachbarn (2002):

Als man mich zum Botschafter machte, war ich geschmeichelt. Und dachte gewissermaßen rehabilitiert zu sein. Endlich auch heimisch anerkannt. Und es war teilweise so. [...] Dagegen aber hat sich der böhmische Teich verändert. In seinem schleimigen Wasser besorgten sich die bislang braven Karpfen Zahnprothesen, um sich in Hechte, ja Haie zu verwandeln. Eine Art beschleunigte Revolution. Und der neuböhmische Fisch, noch immer ein bißchen schlammig und dick, zeigt auch schon Zähnchen ${ }^{14}$.

Diese Erfahrung hinderte Gruša jedoch keineswegs daran, sich auch weiterhin gesellschaftlich zu engagieren und als kultureller Vermittler zu wirken. 1999 bis 2007 beteiligte er sich als Mitherausgeber an der 33-bändigen Ausgabe der Tschechischen Bibliothek, einer Initiative der Robert Bosch Stiftung unter der Schirmherrschaft der Präsidenten der Tschechischen Republik und der Bundesrepublik Deutschland. Im Jahr 1999 erschien seine witzige und höchst geistreiche, inzwischen in mehrere Sprachen übersetzte Gebrauchsanweisung für Tschechien - ein Essayband, in dem nicht nur die Sprache, Kultur, Bräuche und Geschichte Tschechiens vorgestellt werden, sondern auch die Selbstund Fremdbilder (Deutsche/Tschechen) bewusst gemacht, kritisch beleuchtet und auf ihre Haltbarkeit hin überprüft werden: „Das Interessante an Vorurteilen ist nicht ihre Schäbigkeit, sondern die Tatsache ihrer Nützlichkeit. Wären sie nicht für eine Kollektivseele zu gebrau-

14. Jiří Gruša, Glücklich heimatlos. Einblicke und Rückblicke eines tschechischen Nachbarn, Stuttgart, Hohenheim-Verlag, 2002, S. 230. 
chen, sie wären längst überholt ${ }^{15}$.“ - so „lobt“ Jiří Gruša die Vorurteile, die sie in der Folge auseinandernimmt und subversiv unterläuft.

Im Essay fand Gruša zweifelsohne eine besonders geeignete literarische Form für seine scharfsinnig und einfallsreich formulierten Überlegungen und Kritik der gesellschaftlichen Zustände resp. Missstände, teils ironisch, teils humorvoll, jedoch immer ganz genau beobachtet und objektiv analysiert. Seine frühen Texte, Essays, Gedanken, Interviews und Tagebuchnotizen aus den Jahren 1964 bis 2004 wurden unter dem Titel Als ich ein Feuilleton versprach. Handbuch des Dissens und Präsens von Schriftsteller Michael Stavarič ins Deutsche übersetzt mit dem Ziel, „den Ausgangspunkt von Jiří Grušas Schaffen, den ,Kontext"“, zu beleuchten, wie im Vorwort betont wird, denn sie stellen „eine Anleitung dar, wie man sich unter diesen oder jenen Bedingungen verhalten kann, ja soll, was moralisch richtig und menschlich wichtig ist ${ }^{16 "}$. Diese Fragen sowie die Frage nach der Macht der Mächtigen bzw. der (Ohn) Macht der Machtlosen beschäftigten Gruša sein Leben lang, insbesondere im Zusammenhang mit der tschechoslowakischen Geschichte.

Hier galt sein Interesse besonders Tomáš Garrigue Masaryk und Eduard Beneš. Mit der letzteren setzte sich Gruša wiederholt in seinen Vorträgen auseinander, da Beneš als Präsident durch seine Entscheidungen im Jahr 1938 und 1948 die tschechoslowakische und somit auch Grušas eigene (Lebens)Geschichte maßgeblich beeinflusst hatte. 2011 fasste er seine bisherigen Überlegungen zusammen und publizierte sie in erweiterter Form als Essay zunächst auf Tschechisch unter dem Titel Beneš jako Rakušan, ein Jahr danach (bereits nach Grušas Tod) erschien auch die deutsche Version Beneš als Österreicher. In seinem letzten Essay untersucht Gruša an Benešs Beispiel das Phänomen des Defätismus und fragt nach den Ursachen seines Verhaltens, dass zur zweifachen Kapitulation führte - als er widerstandlos die Tschechoslowakei an das Nazi-Regime 1938 übergab und als er 1948 die Machtübernahme der Kommunisten legalisierte. Ebenso ist er auch für die Homogenisierung der nationalen Struktur der Tschechoslowakei nach 1945 durch die gesetzlich verordnete Aussiedlung der deutschsprachigen Bevölkerung (sog. Beneš-Dekrete) verantwortlich:

15. Jiří Gruša, Gebrauchsanweisung für Tchechien. Stuttgart, Hohenheim-Verlag, 1999, S. 16. 16. Michael Stavarič, „Vorwort des Herausgebers“, in Jiři Gruša, Als ich ein Feuilleton versprach. Handbuch des Dissens und Präsens (übersetzt und herausgegeben von M. Stavarič), Wien, Czernin Verlag, 2004, S. 9-13, S. 13. 
Seine [Benešs] Kapitulation mit all ihren Konsequenzen für unsere Familie erlebte ich als zehnjähriger Junge. Mein Vater musste in der Zeit des realexistierenden Sozialismus seinen Lebensunterhalt als Bergmann verdienen und hatte kein gutes Wort für ihn übrig. Schon 1938 als Soldat in einem Betonbunker im Grenzland in Stellung, mißfiel ihm ganz und gar, dass wir kapituliert hatten. Als ich dann 1968 selbst die nächste tschechische Kapitulation verdauen musste, stellte ich mir Vaters Frage: Warum setzen wir uns nicht zur Wehr ${ }^{17}$ ?

Mit diesem Essay, mit Zügen eines Faktenromans (da mit Fakten wissenschaftlich gearbeitet wird, jedoch in literarischer Sprache), sorgte Gruša für eine rege Diskussion vor allem in Tschechien. In Form eines Psychogramms bietet er eine psychologisierende Sicht auf diese historisch umstrittene Persönlichkeit und eröffnet zugleich die Möglichkeit einer neuen Interpretation, einhergehend mit einer kritischen Reflexion des eigenen Selbstbildes. Mit der Wahl eines programmatischen Titels verankert Gruša Beneš bewusst und provokativ nicht im deutschen, sondern im österreichischen kulturgeschichtlichen Kontext:

Warum Beneš als Österreicher? Weil Austria in Beneš’ Psyche eine Art Hassliebe darstellt. Zuerst hatte er eine geniale Vision, wie dieses Konglomerat zu retten gewesen wäre (siehe seine Dijoner Dissertation). Als sie scheiterte, überkam ihn Lust, Österreich zu zerstören. Die Tschechoslowakei zeigte sich aber als Österreich im Kleinformat. Und da wollte er sie „entösterreichern“ und war erstaunt, als sie dazu gleich verschwand ${ }^{18}$.

Die Widersprüchlichkeit von Beneš politischen Taten widerspiegelt sich in der Widersprüchlichkeit des Umgangs mit seinem Bild und Vermächtnis. Trotz der offiziellen Denkmäler, die ihn zelebrieren, überwiegt auf beiden Seiten, wenn aus unterschiedlichen Gründen, das negative Bild - statt eines „Benedictus“ ein „Maledictus“ - ob gerecht oder ungerecht bleibt offen: „Weisheit, Tapferkeit, Gerechtigkeit, Mäßigung. Eduard Beneš kannte sie sicher, legte sie jedoch auf seine Weise aus: Weisheit hielt er für Schlauheit, Tapferkeit für Biegsamkeit, Gerechtigkeit für Vergeltung und Mäßigung für Kälte“19 - lautet Grušas lakonisches Fazit.

17. Jiří Gruša, Im Banne der Kapitulation, Brno, Masarykova univezita, 2011. BenešFestvortrag von Jiří Gruša am 18.4.2011 in Brno zum Anlass der Präsentation seines Essays Beneš jako Rakušan (vorgetragen auf Tschechisch, abgedruckt dreisprachig tschechisch, deutsch und französisch).

18. Ebd.

19. Ebd. 
Wird es uns gelingen, die Folgen dieser geschichtlichen Entscheidungen, die tiefe Wunden im "Gesicht“ Mitteleuropas hinterlassen haben, produktiv umzusetzen und das Heimische mit dem Unheimlichen zu kombinieren? Wie wird die Zukunft Tschechiens, wie die Zukunft Europas aussehen? Wie wird die Rolle Tschechiens in Europa sein? Was für ein Europa wird es sein? Grušas Vision von einem gemeinsamen Europa, die er in einem Gespräch mit Michael Stavarič vor mehr als zehn Jahren formulierte, hat nichts an ihrer Aktualität verloren - im Gegenteil - sie scheint das aktuelle Problem des Projekts „Europa“ genau erfasst und auf den Punkt gebracht zu haben:

Wenn man nicht in Hekatomben universeller Unifizierungen enden will, dann muss man den Weg von ewigen Wahrheiten zu zeitlichen Wahrheitsmodellen finden, von Beschwörungen der Identität zur Ehrfurcht von Komplexität; von Phrasen der großen Vereinigung zur Demut vor kleinen Einheiten. Von der Bibel und anderen Büchern zu sorgfältig geprüften Texten - und Kontexten ${ }^{20}$.

20. Michael Stavarič, „Der Fragebogen oder auch - die 16 Antworten des Herrn Gruša“, in Gruša, Als ich das Feuilleton verprach, S. 248-261, S. 261. 